\title{
Bipolar disorder: an overview
}

\author{
E Bronkhorst, J Motubatse
}

School of Pharmacy, Sefako Makgatho Health Sciences University

Corresponding author, email:elmien.bronkhorst@smu.ac.za

\begin{abstract}
Bipolar disorder (BD) is a chronic disorder characterised by abnormal mood changes and fluctuation in energy levels. The disease is characterised by a depressive episode, which can last up to a few months, and include low energy levels, hypersomnia, cognitive impairments, decreased sexual desire, carbohydrate craving, and weight gain. Conversely, bipolar disorder also has a stage of exhilaration and excitement, which can be classified as a manic episode. The typical presentation of these episodes of mania includes high energy hallucinations and delusions, sleep deprivation, fast speech and a constant need to be active. Risk factors that contribute to development of BD include the use of cannabis, influenza during the third trimester of pregnancy, as well as smoking during pregnancy, the use of cocaine, opioids, tranquilisers, stimulants and sedatives during pregnancy, and regular substance use during a period of a year. Parental loss before the age of five, war trauma and stress, as well as traumatic head injury may influence the condition.
\end{abstract}

Clinical criteria are the mainstay of diagnosis of BD. Differentiating features, which were found to be related to BD, include early age of onset, family history, atypical features and mixed symptoms. Screening tools like the Mood Disorder Questionnaire (MDQ) and Hypomania Checklist (HCL-32) have shown the ability to differentiate BD from major depressive disorder (MDD).

Treatment differs between the different phases of $\mathrm{BD}$, but lithium can be considered as initial monotherapy, since it is effective against manic, depressive and mixed relapses, and has shown better evidence for prevention of new episodes. It is also associated with a reduced risk of suicide and self-harm in BD patients.

Keywords: bipolar disorder, mania, depression, pharmacological management

\section{Introduction}

Major depressive episodes are a prominent symptom in both bipolar disorders (BD) and major depressive disorder (MDD), which is the reason that up to $69 \%$ of patients with $\mathrm{BD}$ are misdiagnosed. ${ }^{1}$ Bipolar disorder (BD), previously known as manic-depressive disorder, is a chronic disorder characterised by abnormal mood changes and fluctuation in energy levels. ${ }^{2}$ The disease is characterised by an individual spiralling into a state of melancholy, which can last up to a few months and is known as a depressive episode. The typical presentation of these episodes of depression includes low energy levels, hypersomnia, cognitive impairments, decreased sexual desire, carbohydrate craving, and weight gain. Suicide attempts are extremely common during such an episode and individuals have confessed to being in a very dark space with no other way to escape. Conversely, bipolar disorder also has a stage of exhilaration and excitement, which can be classified as a manic episode. The typical presentation of these episodes of mania includes high energy hallucinations and delusions, sleep deprivation, fast speech and a constant need to be active. ${ }^{3}$
Bipolar disorder can be divided into a number of sub-types based on the identification of specific mood episodes, namely:

- Bipolar I disorder

- Bipolar II disorder

- Cyclothymic disorder

- Substance/medication induced bipolar and related disorder

- Bipolar and related disorder due to another medical condition

- Other specified bipolar and related disorder

- Unspecified bipolar and related disorder $3,4,5,6$

Individuals with BD type I experience periods of severe mania and short depressive episodes, which usually follow the manic phase directly. It can also present as mixed episodes. Individuals with BD type II experience episodes of hypomania and severe depression, which is regularly misdiagnosed as major depression disorder. ${ }^{1}$ Individuals diagnosed with unipolar disorder present with symptoms of a chronic sub-syndromal depressive state ${ }^{2}$ which is also often misdiagnosed as major depressive disorder. ${ }^{1}$ 


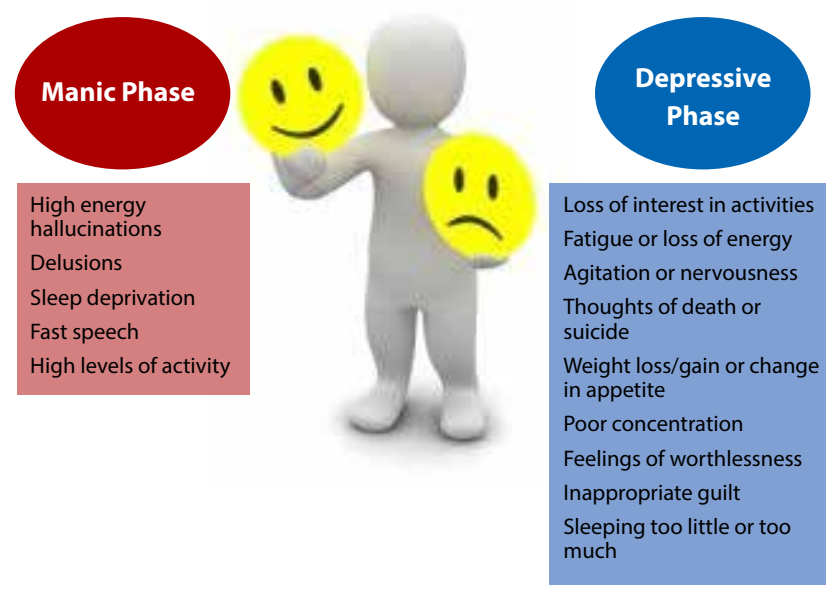

Figure 1: Signs and symptoms of the manic and depressive phases of bipolar disorder

Cyclothymic disorder presents with chronic fluctuations between sub-syndromal depressive and hypomanic episodes. ${ }^{5}$ Longitudinal assessment and detection of hypomanic periods are crucial to differentiate BD from other conditions. ${ }^{2}$

Bipolar Disorder type I is experienced by more than $0.8 \%$ of the world's population, ${ }^{2}$ and around $1.1 \%$ of the population for BD type II.7 This condition does not discriminate in terms of gender, race, nationality, social economy class or level of education. ${ }^{2,8}$ There is an equal distribution in males and females, but males tend to experience the manic phase more commonly and females the depressive phase. Genetic components play a role in the diagnosis of $\mathrm{BD}$, where first-degree relatives with $\mathrm{BD}$ will increase one's chances of suffering the disease. ${ }^{9}$ The usual age of onset is late adolescence and early adulthood, although some cases in children have been reported. In many communities, $\mathrm{BD}$ is not recognised as an illness, hence many people suffer in silence. $^{2}$

\section{Risk factors for developing bipolar disease}

Risk factors that contribute to BD include the use of cannabis, one to four times per week, influenza during the third trimester of pregnancy, as well as smoking during pregnancy, which may affect the foetus. In addition, such risk factors may also include the use of cocaine, the use of opioids, tranquilisers, stimulants and sedatives during pregnancy, and regular substance use during a period of a year. Parental loss before the age of five, war trauma and stress, as well as traumatic head injury may influence the condition. ${ }^{7}$

Factors that may worsen the condition in a diagnosed individual may include the use of alcohol, opioids, tranquilisers, stimulants and sedatives. Anxiety disorders, socio-economic problems, child abuse and sexual maltreatment may also worsen the condition. ${ }^{7}$

\section{Pathophysiology}

Theoretically, BD is the result of an imbalance in neurotransmitters in the brain (serotonin, dopamine and noradrenalin). A dysregulation between the excitatory (noradrenaline (NA), dopamine (DA), glutamate and aspartate) and inhibitory neurotransmitters (serotonin (5-HT) and GABA) has an effect on the normal neuronal transmissions in the brain. Serotonin is seen as an inhibitory neurotransmitter due to the effect it has on decreasing acetylcholine release, where an excess thereof results in depression. ${ }^{10}$ In the monoamine hypothesis, an excess of catecholamines, namely NA and DA, causes mania, while the exact opposite is found when there is a deficit of neurotransmitters, namely NA, DA and $5-\mathrm{HT}$, which will cause depression. $^{5}$

An increase in the concentration of NA in the cortical and thalamic areas of the brain is evident in studies of $B D$ individuals. Noradrenalin and its metabolite 3-methoxy-4hydroxyphenylglycol (MHPG) tend to be lower during the depressive phase and higher during the manic phase of $\mathrm{BD}$. High levels of MHPG have been identified in urine and cerebrospinal fluid during the manic phase of BD. ${ }^{10,11}$

The cholinergic hypothesis states that a deficiency of acetylcholine causes an imbalance in cholinergic-adrenergic activity and can increase the risk of manic episodes, whereas an increased level of acetylcholine activity will result in depressive episodes. ${ }^{5}$

The hypothalamic-pituitary-thyroid axis dysregulation hypothesis states that hyperthyroidism can cause manic-like symptoms and hypothyroidism may cause depression. ${ }^{5}$ Hormonal changes during the female life cycle can cause dysregulation of neurotransmitters (e.g., premenstrual, postpartum, and perimenopause). ${ }^{5}$

Calcium concentrations, both intra- and extra-cellular, may affect the synthesis and release of NA, DA, and 5-HT, as well as the excitability of neuronal firing. Through this mechanism, abnormal calcium and sodium activity can cause neurotransmitter dysregulation, such as hypocalcaemia that has been associated with mood irritability, anxiety, mania and psychosis. Hypercalcaemia has been associated with depression, stupor, and coma. ${ }^{5}$

\section{Diagnosis of BD}

No specific biomarker has been approved for the diagnosis of any mental disorder, including BD. Clinical criteria, including those stipulated in the $5^{\text {th }}$ edition of the Diagnostic and Statistical Manual of Mental Disorders (DSM-5) prevails. ${ }^{2}$ Differentiating features, which were found to be related to $B D$, include early age of onset, family history, atypical features and mixed symptoms. ${ }^{1}$ Screening tools like the Mood Disorder Questionnaire (MDQ) and Hypomania Checklist (HCL-32) have shown the ability to differentiate BD from MDD. ${ }^{1}$

\section{Management of bipolar disorder}

The third version of the guidelines for the scope and targets of treatment for bipolar disorder was updated in 2016 by the British Association for Psychopharmacology (BAP). These guidelines include the diagnosis of $\mathrm{BD}$, clinical management and the use of medication in the short-term treatment of episodes, as well as to prevent relapse and to discontinue treatment. ${ }^{12}$ 


\section{Fundamentals of patient management}

The following principles apply:

1. Hypomania, mania and depression should be accurately diagnosed since the individual episodes may display mixed features of the opposite pole ${ }^{12}$ and the depressive phase may often be misdiagnosed as major depression.

2. In bipolar patients, early intervention is highly desirable, especially in the management of young people. During the manic phase of bipolar depression, admission to a specialised care facility should be considered ${ }^{12}$ to ensure the safety of the patient as well as others close to him.

3. The physician should take responsibility for the diagnosis and physical examination, and to establish and maintain a therapeutic alliance with the patient by explanation of the medical plan of treatment. The patient and his or her family must be educated about the disorder, and the different experiences and beliefs about the disease must be considered. Particularly in younger patients, treatment adherence is often poor, and counselling on treatment adherence is considered an important part of the treatment plan. Awareness of stressors like sleep disturbances, which can trigger manic episodes, early signs of relapse and regular patterns of activity should be highlighted. Poor outcomes are also associated with alcohol and drug use and should be appropriately managed. Functional recovery seldom occurs within 12 weeks following remission of mood symptoms and the patient should evaluate and manage any functional impairments. Patients should also be discouraged to make major life decisions while in a manic or depressive state. Cardiovascular disease can play an important role in premature mortality of bipolar disease patients. Thus the patient's physical health should be considered during clinical assessment, taking weight and other relevant risk factors into consideration. The incidence of risks like self-harm, suicide, victimisation, violence and criminality is increased in bipolar patients and should also be considered. ${ }^{12}$

\section{Non-pharmacological management}

Electroconvulsive therapy (ECT) may be considered for manic patients who are severely ill, or who are experiencing treatmentresistant mania. ${ }^{12}$ It may also be considered in cases of delirious mania, since it can be seen as a medical emergency when accompanied by fever, dehydration and autonomic dysfunction.

Psychotherapy currently provides no alternative strategy for the management of BD. ${ }^{12}$ If adults with $B D$ are offered a psychological intervention, it must be developed specifically for bipolar disorder with a published, evidence-based manual describing how it should be delivered. Monitor the patients' mood for signs of mania or hypomania during psychotherapy. ${ }^{13}$ Cognitive behavioural therapy has not consistently been found to be beneficial. ${ }^{8}$

\section{Pharmacological treatment}

Treatment differs between some of the different phases of BD, as highlighted below.

\section{Acute manic phase}

Patients experiencing acute mania will require short-term treatment in an appropriate clinical setting. For patients with severe manic episodes not already taking long-term treatment for bipolar disorder, the administration of an oral dopamine antagonist is advised..$^{12}$ Haloperidol, olanzapine, risperidone and quetiapine are effective in providing a short-term reduction of symptoms. ${ }^{12}$ Valproate may be an alternative with a decreased risk of adverse motor reactions, but is not considered an option in women of child-bearing age. Aripiprazole, carbamazepine and lithium are alternative options. ${ }^{12}$

Where severely agitated patients require parenteral treatment, dopamine antagonists/partial agonists and GABA modulators, like the benzodiazepines, must be used at the lowest dosages necessary to alleviate the symptoms in question. ${ }^{12}$ The dosage of dopamine antagonists should not be increased to obtain a sedative effect. To promote sleep in agitated patients, in the short-term, GABA modulating drugs must be considered. ${ }^{12,13}$ Treatment selection should be guided by the patient's previous experiences and preferences. During manic episodes, antidepressant drugs (used for unipolar depression) should be tapered and discontinued. Once successful treatment has been initiated, long-term treatment can be considered. ${ }^{12,13}$

For patients experiencing a manic episode while taking longterm treatment, the highest tolerated dosage of the current treatment must be offered. Increasing the dosage of the dopamine antagonists or valproate may be sufficient to control manic symptoms. If the patient is being treated with lithium, the serum concentration must be monitored, and needs to be within the usual target range $(0.6-0.8 \mathrm{mmol} / \mathrm{L})$; higher dosages may be beneficial, but carry a greater risk of harm. ${ }^{12}$ For patients on lithium therapy, a dopamine antagonist or valproate may be considered as an additional treatment option. ${ }^{12}$

Symptoms that are inadequately controlled with optimised dosages of the first-line treatment options may occur because of poor adherence, or due to the contributions of substance use or other medication. In these cases, intense counselling and medically-assisted withdrawal is required. ${ }^{12}$

\section{Acute depressive episodes}

Uncertainty and difference of opinion still exist over the option of choosing anti-depressants during the depressive phase of BD. For patients diagnosed with a first depressive episode or not already taking long-term treatment for their bipolar disorder, dopamine antagonists like quetiapine (most convincing shortterm-efficacy and relapse prevention profile), lurasidone or olanzapine have the advantage of treating manic phases as well. ${ }^{12}$

Antidepressants have not been adequately studied in bipolar disorder, and when considered in the depressive phase, should be in combination with a drug for mania (dopamine antagonists, valproate or lithium). 


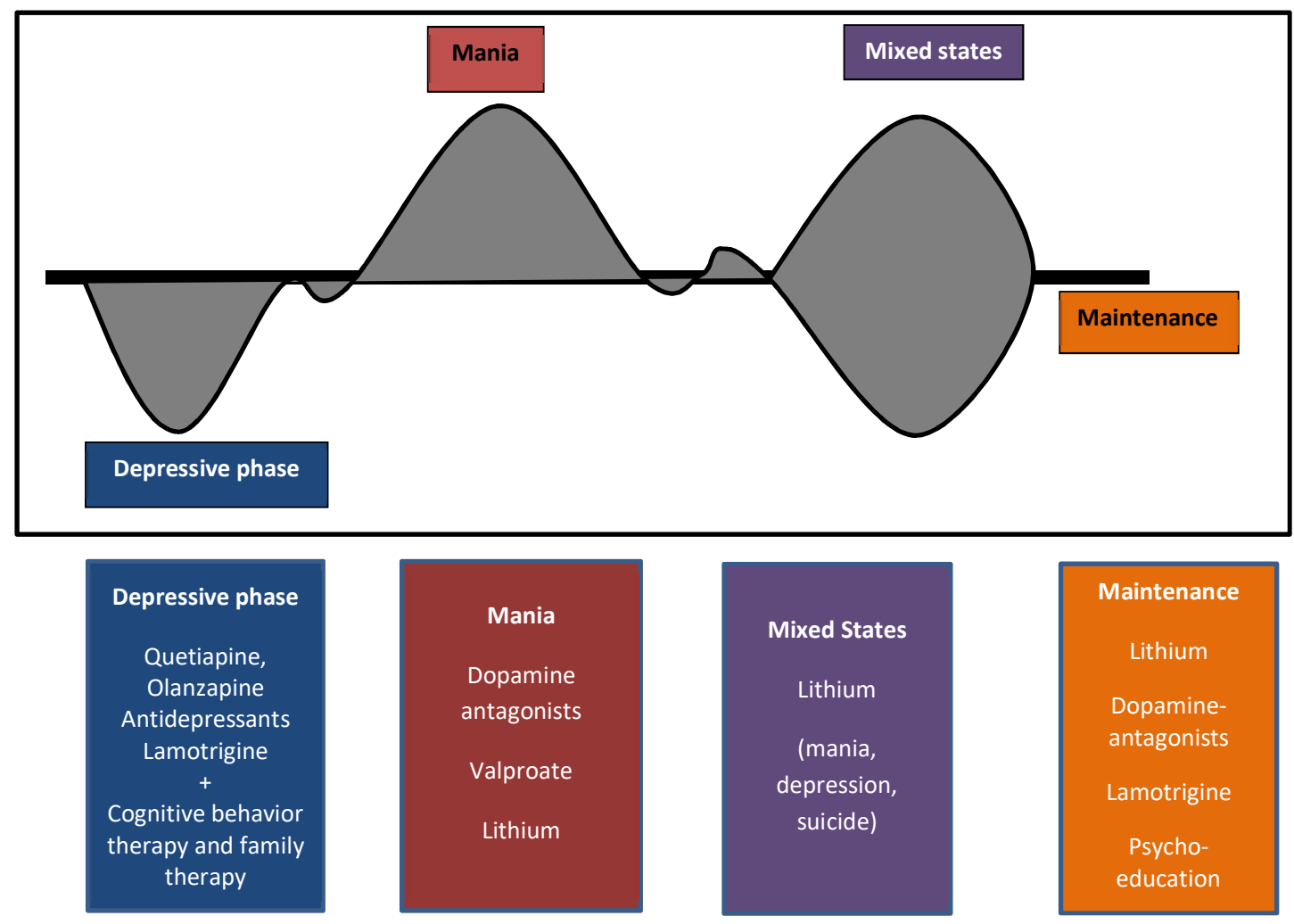

Figure 2: Treatment during the different phases of bipolar illness

Initial treatment with lamotrigine, with incremental dosing, can be considered in addition to agents preventing the recurrence of mania. ${ }^{12}$ Electro-convulsive therapy may be considered for patients with high suicidal risk or treatment resistance. ${ }^{12}$

For patients suffering a depressive episode while on long-term treatment, ensure that the current treatment will protect the patient from manic relapse. Ensure adequate doses and optimum serum concentration of lithium. If dosages are optimised and a patient fails to respond, or the depressive symptoms are significant, additional treatment (as for a new depressive episode diagnosis) should be initiated. ${ }^{12}$

Monotherapy with antidepressants is not recommended, as it may precipitate a switch to mania or mood instability, especially the monoamine re-uptake inhibitors (venlafaxine, duloxetine, amitriptyline and imipramine). Singe-action drugs, like the selective serotonin re-uptake inhibitors, show slightly less risk, especially if used in combination with a drug for mania. ${ }^{12}$

Very little information is available on the treatment of resistant depression in BD (failure to respond to an antidepressant and quetiapine, olanzapine, lurasidone and lamotrigine, singly and in combination), but adequate anti-manic cover with lithium, valproate or dopamine antagonists will be necessary. Electroconvulsive therapy may be an option. ${ }^{12}$

After successful initiation of treatment for de novo depression, long-term treatment should be considered.
Long-term treatment (maintenance)

The long-term treatment of BD should include a wider package of treatment, including psycho-education, motivational and family support, and acceptance of treatment by the patient. Without active acceptance of the need for long-term treatment, adherence may be poor. ${ }^{12}$

The preferred strategy for oral medicines to prevent new mood episodes is for continuous rather than intermittent treatment. Randomised controlled trials with relapse prevention designs only supports the efficacy of limited individual medicine with lithium, olanzapine, quetiapine, risperidone and to a lesser extent, valproate, for the prevention of manic relapse, while only lamotrigine, lithium and quetiapine were convincingly shown to prevent depressive relapse. ${ }^{12}$

The relevant rates, according to hospital admission data, for patients on and off treatment over a period of four years, support the efficacy of the following agents, in decreasing order of effectiveness:

- Lithium

- Valproate

- Olanzapine

- Lamotrigine

- Quetiapine

- Carbamazepine.

Lithium can be considered as initial monotherapy, ${ }^{12,13}$ since it is effective against manic, depressive and mixed relapses, and has shown better evidence for prevention of new episodes. It is also 
associated with a reduced risk of suicide and self-harm in $\mathrm{BD}$ patients. ${ }^{12}$

The standard of care that patients on lithium therapy should receive includes the monitoring of plasma lithium concentrations, with a target range of $0.6-0.8 \mathrm{mmol} / \mathrm{L} .{ }^{12}$ Lithium plasma concentration monitoring is recommended at threemonthly intervals for the first year of treatment, followed by sixmonthly thereafter. ${ }^{13}$

At the neurotransmitter level, lithium modulates dopamine, glutamate and GABA neurotransmission. Conversely, at the intracellular signalling level, lithium inhibits proteins such as PKC, MARCKS, GSK-3, IPPase (inositol poliphosphatase) and IMPase (inositol mono-phosphatase).

\section{Discontinuation of long-term treatment}

Any treatment for BD disease should be tapered over a period of at least four weeks, longer if possible. An early risk of abrupt lithium discontinuation is a relapse to mania. Even though medication can be discontinued, a management plan to recognise and treat early warning signs of future relapse to mania or depression will be necessary, and the short-term care and regular monitoring of patients will still be required. ${ }^{12}$

\section{Conclusion}

Bipolar disorder (BD), previously known as manic depressive disorder, is a chronic disorder characterised by abnormal mood changes. The disease is characterised by depressive episodes during which suicide attempts are extremely common. A stage of exhilaration and excitement, which can be classified as a manic episode will typically present with high energy hallucinations and delusions, sleep deprivation, fast speech and constant need to be active.

Electroconvulsive therapy is the only non-pharmacological treatment that may be considered for manic patients who are severely ill, or who are experiencing treatment resistant mania. For patients with severe manic symptoms, the recommended treatment includes lithium, valproate and the dopamine antagonists, or lithium in combination with valproate or the dopamine antagonists. The depressive stage of BD depression should not be treated with monotherapy of anti-depressants, and dopamine antagonists, valproate or lithium should be continued to prevent the precipitation of a manic attack. Single action anti-depressives like the serotonin re-uptake inhibitors are recommended in combination with a drug for mania. Lithium still shows the strongest evidence as a mood stabilizer preventing relapse to mania and depression.

\section{References}

1. Chen X, Xu G, Dang Y, Ouyang H, Guo Y, Aksiskal HS, Lin K. Differentiating bipolar type I and II depression from unipolar depression: the role of clinical features, current symptoms and a past hypomanic symptoms checklist. Neuropsychiatry (London). 2016;6(4):167-77.

2. Grande I, Berk M, Birmaher B, Vieta E. Bipolar disorder. The Lancet. 15 Apr 2016;387(10027): 1561-72.

3. Brenner GM, Stevens CW. Pharmacology. $4^{\text {th }}$ edition, Chapter 22: Psychotherapeutic Drugs. Philadelphia: Saunders-Elsevier Company.

4. Lojko D, Suwalska A, Rybakowski J. Bipolar and related disorders and depressive disorders in DSM-5. Psychiatria Polska. 2014; 48(2): 245-60.

5. Drayton SJ, Pelic DM. Bipolar Disorder in Di Piro et al. Pharmacotherapy: a Pathophysiological approach. $9^{\text {th }}$ edition. NewYork: McGraw-Hill. 2014.

6. DSM-5 Update. Supplement to Diagnostic and statistical manual of mental disorders. $5^{\text {th }}$ Edition. 2015. Available from: http://passthrough.fw-notify. net/download/617962/http://dsm.psychiatryonline.org/pb-assets/ dsm/update/DSM5Update2015.pdf

7. Marangoni $C$, Hernandez M, Faedda GL. The role of environmental exposures as risk factors for bipolar disorder: a systematic review of longitudinal studies. J Affect Disord. Mar 2016 15;193:165-74.

8. De Crescenzo F, Economou A, Shrpley AL, Gormez A, Quested DJ. Actigraphic features of bipolar disorder: A systematic review and meta-analysis. Sleep Med Rev. 2016; 1-12.

9. Meier SM, Uher R, Mors O, Dalsgaard S, Munk-Olsen T, Laursen TM, et al. Specific anxiety disorders and subsequent risk for bipolar disorder: a nationwide study. World Psychiatry. 1 Jun 2016;15(2):187-8.

10. Tsatsaris A, Domenikos S, Psychos C, Moutsiounas D. Chaos theory and behavioural patterns: a theoretical approach to psychosis, bipolar disorders and depression. Journal of Advanced Biotechnology and Bioengineering. 2016;4(1).

11. Hadaeghi F, Golpayegani M, Jafari S, Murray G. Toward a complex system understanding of bipolar disorder: a chaotic model of abnormal circadian activity rhythms in euthymic bipolar disorder. Aust N Z J Psychiatry. 2016;50(8): 783-92.

12. Goodwin GM, Haddad PM, Ferrier IN, Aronson JK, Barnes TRH, Cipriani A, et al. Evidence-based guidelines for treating bipolar disorder: revised third edition Recommendations from the British Association for Psychopharmacology. J Psychopharmacol. 2016;30(6):495-553.

13. National Institute for Health and Care Excellence (NICE). Bipolar Disorder: Assessment and Management. 2014. Available from: https://www.nice.org.uk/ guidance/cg185 